\title{
A FISIOTERAPIA NO TRATAMENTO DA FADIGA NOS PACIENTES DE ESCLEROSE MÚLTIPLA
}

\section{PHYSIOTHERAPY IN THE TREATMENT OF FATIGUE IN MULTIPLE SCLEROSIS PATIENTS}

\section{CARINA SOUSA SANTOS}

Acadêmica do 9o período do Curso de Fisioterapia. Universidade Presidente Antônio Carlos - ALFAUNIPAC de Teófilo Otoni/MG -

E-mail: carinassantos19@gmail.com

\section{MARILENE MENDES GONÇALVES}

Acadêmica do 9o período do Curso de Fisioterapia. Universidade Presidente Antônio Carlos - ALFAUNIPAC de Teófilo Otoni/MG -

E-mail: marilene.goncalves10@yahoo.com.br

\section{REJANE GOECKING BATISTA PEREIRA}

Professora Orientadora. Especialista em Fisioterapia Neurológica pela UFMG Especialista em Terapia Intensiva Neonatal pela Escola de Saúde Pública - MG Fisioterapeuta Responsável Técnica Unimed Três Vales. Professora do Curso de Fisioterapia da Universidade Presidente Antônio Carlos campus Teófilo Otoni/MG - BRASIL.

E-mail: rejanegoecking@hotmail

Recebido: 16/06/2021 - Aceito: 17/06/2021

\section{Resumo}

A Esclerose múltipla é uma doença autoimune, progressiva crônica que atinge o SNC, levando a lesões no cérebro, medula, e bainha de mielina atrapalhando a condução nervosa, tendo sua etiologia desconhecida e sua maior incidência em mulheres com idade entre 20 e 50 anos. Devido essas lesões no SNC, pessoas com EM desenvolve sintomas variados, tem em comum a fadiga sendo em geral a principal queixa. Essa revisão bibliográfica teve como intuito mostrar e relatar o impacto positivo de como a fisioterapia pode ajudar na fadiga de pessoas com EM. O presente 


\title{
$2021 / 02$
}

\section{ISSN 2178-6925}

estudo caracteriza-se como qualitativo por meio de uma analise bibliográfica estruturada na denominação de revisão de literatura, foi realizada uma busca na base de dados do Google acadêmico, PUBMED e SCIELO, foram limitadas entre os anos 2011 e 2021. Com base nesse estudo de revisão bibliográfico, conclui-se que a fadiga em pessoas com Esclerose múltipla é uma dos sintomas mais incomodo, e que estudos de casos mais atuais sobre técnicas da fisioterapia trazem resultados satisfatórios no tratamento da fadiga de pessoas com EM.

Palavras- chaves: Esclerose múltipla, reabilitação, fadiga e fisioterapia.

\begin{abstract}
Multiple sclerosis is an autoimmune, chronic progressive disease that affects the CNS, leading to lesions in the brain, spinal cord, and myelin sheath, impairing nerve conduction, its etiology being unknown and its higher incidence in women aged between 20 and 50 years. Due to these CNS lesions, people with MS develop varied symptoms, fatigue being in common and being the main complaint in general. This literature review aimed to show and report the positive impact of how physical therapy can help with fatigue in people with MS. This study is characterized as qualitative through a literature review structured in the name of literature review, a search was performed in the academic Google database, PUBMED and SCIELO, limited between 2011 and 2021. Based on this literature review study, it is concluded that fatigue in people with MS is one of the most bothersome symptoms, and that more current case studies on physical therapy techniques bring satisfactory results in the treatment of fatigue in people with MS.
\end{abstract}

Keywords: Multiple sclerosis, rehabilitation, fatigue and physiotherapy.

\section{INTRODUÇÃO}

A esclerose múltipla (EM) é uma doença autoimune, progressiva crônica que atinge o sistema nervoso central (SNC), levando a lesões no cérebro, medula e na bainha de mielina, ou seja, ocorre uma desmielinização atrapalhando a condução nervosa, tendo a sua etiologia desconhecida tem sua maior incidência em mulheres de raça branca e com idade entre 20 e 50 anos.

O diagnóstico de EM é essencialmente clinico, baseia-se na anamnese, achados clínicos e testes laboratoriais de suporte na avaliação dos sintomas percebidos e relatados pelo paciente, juntamente com exames de ressonância magnética, tomografia computadorizada e exame do líquido cefalorraquidiano.

Devido às lesões no sistema nervoso causadas por essa patologia, as pessoas com EM desenvolvem sintomas variados, tendo em comum em sua maioria a fadiga, de uma forma geral é a principal queixa dos indivíduos com EM ocorrendo em aproximadamente $80 \%$ desses indivíduos, com isso acaba que atrapalha a realização atividades simples de vida diária, sendo a fadiga dividida na EM dois tipos: fadiga central (primária) que está diretamente ligada aos fatores da 


\section{$2021 / 02$}

\section{ISSN 2178-6925}

evolução da EM e fadiga periférica (secundária) que é acarretada devido a outras consequências patológicas da doença. A fadiga física é definida como a sensação de exaustão presente durante ou após a realização de atividade de vida diária ou exercício físico. Ao citar EM é importante saber das dificuldades encontradas pelos pacientes e familiares durante o processo de identificação e diagnóstico, muitos pacientes passam por diversos exames antes que consigam receber um diagnóstico adequado.

Ao se tratar de uma doença que não tem cura, mas tem tratamento, a fisioterapia se destaca por ser é um dos tratamentos essenciais que pode auxiliar tanto na prevenção da piora da fadiga ou até mesmo quando já está prejudicando de forma direta e negativamente as atividades de vida diária dessas pessoas, com essa revisão foi analisado que um bom condicionamento físico que pode ser conseguido com a fisioterapia tem uma melhora significativa da fadiga.

Essa revisão bibliográfica teve como intuito demostrar o impacto da fisioterapia na fadiga dos pacientes com esclerose múltipla, demonstrando assim a importância em começar o tratamento fisioterapêutico o quanto antes, com isso analisando protocolos mais utilizados atualmente durante o tratamento referente a fadiga em pessoas com EM.

O presente estudo caracteriza-se como qualitativo por meio de uma análise bibliográfica estruturada na denominação de revisão de literatura. Foi realizada uma revisão criteriosa utilizando a literatura disponível, tais como artigos acadêmicos, revistas cientificas, considerando publicações disponíveis nas bases de dados Google acadêmico, PUBMED e SCIELO (Scientific Eletronic Library) foram limitadas entre os anos de no período de 2011 a 2021. As palavras chave utilizadas foram Esclerose Múltipla, Reabilitação e Fadiga. Após essa análise prévia foram lidos diversos artigos para a leitura na íntegra, onde finalmente foram selecionados artigos dos últimos 10 anos que mais se enquadravam no assunto. Para integralizar, foi realizada uma investigação manual nas listas de referências dos artigos selecionados.

Para o critério de inclusão foram utilizados artigos de revisão de literatura e ensaios clínicos que abordavam a fisioterapia na melhora da fadiga desses 


\section{$2021 / 02$}

\section{ISSN 2178-6925}

indivíduos com EM não levando tanto em consideração tratamentos medicamentosos, sendo descartados artigos que fugiam do tema apresentado.

\section{REVISÃO DE BIBLIOGRÁFICA}

\subsection{Esclerose múltipla}

A esclerose múltipla é uma patologia que atinge o sistema nervoso central, uma doença crônica autoimune, ou seja, as células de defesa ataca o próprio sistema nervoso que vem a causar lesões no cérebro e medula, desmielinização da bainha de mielina, tendo sua etiologia ainda desconhecida. Essa perda de mielina na esclerose múltipla atrapalha a condução dos impulsos nervosos, tendo maior incidência dos jovens sendo mais comum em mulheres (ATUDILLA, P. et al (2011)).

Existem três tipos de esclerose múltipla com evoluções diferentes, Esclerose Múltipla Remitente Recorrente (EMRR) que é a mais típica entre esses pacientes que ocorrem surtos e remissões, e períodos com melhora parcial ou completa dos sintomas. Esclerose Múltipla Secundária Progressiva (EMSP) que decorre de uma evolução da EMRR, sua característica é que depois de alguns surtos e remissões, o paciente entra em uma fase progressiva não sendo necessário mais ter surtos para ter os sintomas ocorrendo de uma forma gradativa. Esclerose Múltipla Primária Progressiva (EMPP) ocorre de uma piora gradual dos sintomas sendo mais raro entre os pacientes. Citado por Festugato, N. et al pág. 3. 2019 "Quanto aos tipos existe três classificações para a EM: EMRR que é o tipo mais comum, em que o paciente apresenta surtos que duram mais de 24 horas; a EMPP, em que o paciente não apresenta surtos, mas sintomas que podem resultar em sequelas; e a EMSP, onde o paciente apresenta surtos no início e, lentamente com o tempo ocorre à piora, sem a ocorrência de surtos".

Os sintomas da esclerose múltipla vão depender de onde o cérebro a medula foi afetado, lembrando que cada pessoa com esclerose múltipla evolui de uma forma diferente, alguns dos sintomas são: fadiga sendo a principal queixa desses pacientes, problema de memória, visão dupla, fraqueza muscular, falta de equilíbrio, sensação de formigamento, parestesia e espasticidade (MESQUITA, B., 


\section{$2021 / 02$}

\section{ISSN 2178-6925}

2013).

\subsection{Fadiga}

A fadiga sendo um sintoma subjetivo vem a ser definida como sensação de cansaço físico e/ou mental profundo, com perda de energias. No geral a fadiga pode ser física relacionada à sensação de corpo pesado, problemas com movimentação, até mesmo ao realizar ABVD's, fadiga mental que associa a dificuldades em manter o foco, de pensar e a fadiga visual como dor nos olhos, ardência levando a uma piora visual. Para estudos e analise da fadiga geralmente são utilizadas a Escala de Severidade da Fadiga (Fatigue Severity Scale - FSS), a Escala Modificada de Impacto da Fadiga (Modified Fatigue Impact Scale-MFIS) e a escala visual analógica para fadiga, que são instrumentos já usados e validados para essas pessoas com EM como citado por MESQUITA, B.S. 2013.

Abordado por COSTA, K.B. et al. (2013) A fadiga é um sintoma subjetivo e pode ser definida como sensação de cansaço físico e/ou mental profundo, com perda de energia. Pode se manifestar a qualquer hora do dia, incluindo ao repouso e estar presente em todas as formas clínicas. A fadiga é relatada como um dos sintomas mais comum e persistente em pessoas com EM e muitas vezes é o sintoma de maior impacto na saúde e sobre a qualidade de vida desses pacientes, a maioria das pessoas com EM vai experimentar fadiga em algum momento durante o curso de sua doença.

Segundo ARAÚJJO S, M.L. et al. (2019), O A fadiga na EM pode ser dividida em dois tipos: primária e secundária; a fadiga primária é causada por fatores relacionados ao processo da doença (fadiga central), enquanto que a fadiga secundária é causada por algumas das consequências patológicas da doença (fadiga periférica). Clinicamente é difícil diferenciar a fadiga primária da secundária, pois as manifestações são semelhantes, portanto a fadiga pode ser multifatorial.

A fadiga tem suas particularidades e subjetividades de pessoas para pessoas com EM, que vem acarretar um desconforto físico e mental para essas pessoas podendo ser muito debilitante para a realização de atividades simples do cotidiano, na qual a fadiga é avaliada por critérios seguindo escalas que foram criadas e adaptadas para melhor entender e classificá-la como já mencionado por 


\section{$2021 / 02$}

\section{ISSN 2178-6925}

MESQUITA, B.S., 2013. As dificuldades em classificar e diferenciar a fadiga parte de que mesmo sendo dividida em fadiga central e periférica, suas manifestações são muitos semelhantes em indivíduos com EM colocando assim a fadiga como um sintoma acarretado por vários fatores, que pode acontecer em qualquer momento do dia sendo por vez manifestada na maioria das pessoas com EM como relatado por COSTA, K.B. et al., 2013 e ARAUJO, S.M.L. et al., 2019.

\subsection{Fisioterapia na fadiga da esclerose múltipla}

A fisioterapia no âmbito geral tem o objetivo de ter a melhora das limitações causadas pela doença até mesmo de forma preventiva desde que tenha o diagnóstico de EM, para preparar de tal forma o corpo para algum agravamento ou de seus surtos, com isso levar o a sua independência em realizar suas atividades e melhorar a qualidade de vida. O tratamento fisioterapêutico parte de uma boa avaliação para traçar um plano de tratamento que respeite a limitação do paciente, tratando desde o problema de movimentar à fadiga, pois pelas observações o aumento da fadiga leva ao medo e insegurança de realizar suas atividades. Independentes do tipo clínico da EM os pacientes geralmente são encaminhados para a fisioterapia quando já perderam sua capacidade de realizar atividades funcionais, ou parte dela, em um ponto em que a doença já provocou danos irreversíveis ao SNC (SCHIWE, D. et al., 2015)

Segundo GOMES, V.T. et al. (2020) . "Dentre os sintomas da EM a fadiga é um dos mais frequentes, podendo ser muito debilitante. Dessa forma, atividades simples do cotidiano podem se tornar mais cansativas e difíceis de serem realizadas. Portanto, exercícios moderados que conservam energia, uso de dispositivos de ajuda, simplificação do trabalho e resfriamento é necessário, já que podem ser eficazes para amenizar a clínica de fadiga do paciente. Na fisioterapia as limitações impostas pela doença são trabalhadas para que seja melhorada a capacidade funcional e como consequência possibilitar a qualidade de vida e precaver complicações debilitantes".

PINA C.N.S.M. (2019) relata que "Uma vez que é impossível prever a progressão desta patologia, devido aos variados e complexos fatores que interferem na mesma, o fisioterapeuta deve atuar precocemente, delimitando 


\section{$2021 / 02$}

\section{ISSN 2178-6925}

objetivos conscientes. Entre estes, promoção de boa qualidade de movimento, execução de tarefas motoras, suporte psicológico com o paciente e seus familiares/amigos, garantir mobilidade articular, estabilidade postural, integridade musculoesquelética, tônus normalizado, redução da fadiga e de contraturas que possam ter surgido devido à espasticidade e diminuição de outras complicações que possam surgir".

PEREIRA, G.C. et al. (2012) "A FNP nesse caso irá fornece subsídios necessários para auxiliar na habilidade do paciente em mover-se ou permanecer estável, guiar o movimento com a utilização de contatos manuais adequados e de resistência apropriada, obter coordenação motora e sincronismo e aumentar a histamina do paciente, evitando a fadiga muscular. Através dos padrões diagonais (geralmente útil para reduzir o tônus) e das ações combinadas dos principais grupos musculares sinergistas (útil quando o paciente se cansa facilmente), a FNP permite a ativação muscular com um gasto menor de energia, o que vai de encontro com nossos achados.

De acordo com o estudo de PEREIRA, G.C. et al. (2012) "A intervenção fisioterapêutica em longo prazo com média de oito horas por mês, durante 18 meses consecutivos para indivíduos com EM surto-remissão promove melhoras significativas na função geral em relação aos que recebem menos fisioterapia. Em nosso trabalho, observamos de uma forma geral, uma melhora das pacientes com apenas quatro horas por mês, em um período de 8 meses quando comparamos os resultados entre as três Avaliações".

Mesmo com poucos estudos que retratam 0 tratamento com eletroestimulação na fadiga em indivíduos com EM, um estudo feito por CHANG, Ya-Ju et al. (2011) quantificou o peso da fadiga central vs fadiga periférica em pacientes com esclerose múltipla, na qual demonstrou que a fadiga central teve mais peso que a fadiga periférica. No estudo também relatou que as 8 semanas de treinamento FES da superfície extensora do joelho teve aumento na resistência à fadiga geral e resistência à fadiga central dos extensores do joelho em indivíduos com EM. A percepção da fadiga medida pelo MFIS também melhorou após o treinamento e também relata que a resistência à fadiga central foi o principal 


\section{$2021 / 02$}

\section{ISSN 2178-6925}

contribuinte para a melhora na resistência à fadiga geral após o treinamento FES de superfície. $O$ dados dessa pesquisa trouxeram resultados satisfatórios para a utilização do FES "FI aumentou de $66,58 \pm 13,00$ por cento para $74,86 \pm 11,02$ por cento ( $\mathrm{df}=6, \mathrm{t}=-3,77, \mathrm{p}<0,01$ ) após 8 semanas de treinamento de superfície FES. Este resultado sugere que a resistência geral à fadiga dos pacientes melhorou. O CFI aumentou de 73,23 \pm 18,11 por cento para 84,49 \pm 11,90 por cento ( $\mathrm{df}=6, \mathrm{t}=-3,36, \mathrm{p}=0,02)$ após 8 semanas de treinamento de superfície FES. Este resultado sugere que 8 semanas de treinamento de superfície FES foi eficaz no alívio da fadiga central dos pacientes. Após 8 semanas de treinamento de superfície FES, o PFI médio mudou de 93,86 \pm 7,36 por cento para 104,80 \pm 9,22 por cento, mas esta mudança não foi estatisticamente significativa ( $\mathrm{df}=6, \mathrm{t}=$ $2,13 p=0,08)$." Com tais dados é notável que em ralação a fadiga periférica a fadiga central é maior tanto antes quanto após o treinamento.

Em um ensaio clínico utilizou hidroterapia com exercícios específico Ai-Chi sendo conduzido por um fisioterapeuta em pessoas com Esclerose múltipla, onde foi dividido em dois grupos, observando assim que o grupo experimental mostrou uma redução significativa na fadiga nas semanas $20(P<0,043)$ e semana $24(P<$ 0,046). O grupo de controle não apresentou dificuldade significativa contra linha de base em qualquer ponto de tempo, com 12\% evidenciando uma melhora. Portanto de acordo com o resultado os a Hidroterapia com exercícios ai-chi não só demonstrou que atua na melhora articular, física, mas também na melhora da fadiga em pessoas com EM (CASTRO-SÁNCHEZ, A.M. et al., 2011).

Como foi mostrado SHIWE, D. et al., BARRETO, M.D. et al., GOMES, T.V et al., PINA, C.N.S.M., PEREIRA, G.C et al, a Fisioterapia com um protocolo de tratamento bem elaborado vem a proporcionar melhora na fadiga juntamente com outros agravos da EM. Nos estudos realizados por CHANG Ya-Ju et al., CASTROSÁNCHEZ, A.M. et al., a utilização de técnicas especificas e individuais de cada estudo demonstram que a uma melhora significativa da fadiga nos indivíduos com EM, para a realização desses ensaios clínicos foram utilizado escalas especificas para avaliação da fadiga. Para BARRETO, M.D. et al. (2018) O trabalho durante o tratamento fisioterapêutico em se ganhar força muscular ajuda no equilíbrio, 


\section{1/02}

\section{ISSN 2178-6925}

marcha consequentemente diminui a fadiga.

\section{CONSIDERAÇÕES FINAIS}

Com base nesse estudo de revisão bibliográfica, conclui-se que a fadiga em pessoas com EM é uma dos sintomas mais incomodo durante desenvolvimento da doença e que atinge aproximadamente $80 \%$ de pessoas com EM, e que na maioria dos casos esses pacientes demoram ir buscar o tratamento fisioterapêutico adequado para evitar a progressão da fadiga.

Com este estudo a fisioterapia é um caminho tanto para a prevenção do aumento da fadiga desde quando tem- se diagnóstico positivo de EM, e mesmo quando a fadiga já está de uma forma mais agressiva nesses indivíduos.

Portanto a fisioterapia se baseia não só no tratamento músculo esquelético de pessoas com EM, mas é fundamental no tratamento da fadiga desde que tenha um protocolo adequado e traçado, e que estudos de casos mais atuais sobre técnicas da fisioterapia trazem resultados satisfatórios no tratamento da fadiga de pessoas com EM.

\section{REFERÊNCIAS}

\section{ALCÂNTARA, L. G. Efeito do treinamento em circuito orientado a tarefas e do} treinamento aeróbico sobre a fadiga em indivíduos com esclerose múltipla: uma revisão crítica de literatura. Monografia: Luciana Gomes de Alcântara - Belo Horizonte. Bibliografia: pag.20-22, 2019. Disponível em:

https://repositorio.ufmg.br/bitstream/1843/30856/1/TCC\%20FINAL.pdf. Acesso em: 05 mar. 2021

ARAÚJO, S.M.L. et al. Tratamentos fisioterapêuticos utilizados para fadiga em pacientes com esclerose múltipla. ceafi.edu.br-2019. Goiás, pag.17, 2019. Disponível em: https:/ceafi.edu.br/site/wp-content/uploads/2019/05/tratamentosfisioteraputicos-utilizados-para-fadiga-em-pacientes-com-esclerose-mltipla.pdf. Acesso em: 30 mar. 2021

ASTUDILLA, P. et al. Relação entre Fadiga e Qualidade de Vida em Pacientes com 


\section{$2021 / 02$}

\section{ISSN 2178-6925}

Esclerose Múltipla: Uma Revisão de Literatura. Revista Neurociências, São Paulo, vol.19 n.3, pag.525-529, 2011. Disponível em:

https://doi.org/10.34024/rnc.2011.v19.8352. Acesso em: 08 mar. 2021

BARRETO, M.D. et al. Esclerose múltipla: considerações gerais e abordagem fisioterapêutica. Arquivo Neuro-Psiquiatria. Recife, vol.67, n.6, pag.908-1014, 2018. Disponível em:

http://www.pergamum.univale.br/pergamum/tcc/Esclerosemultiplaconsideracoesger aiseabordagemfisioterapeutica.pdf. Acesso em: 16 mar. 2021

$\mathrm{BROCH}$, L. et al. High prevalence of fatigue in contemporary patients with multiple sclerosis. Multiple Sclerosis Journal-Experimental, Translational and Clinical, vol.7, n.1, pag.2055217321999826, 2021. Disponível em:

https://doi.org/10.1177/2055217321999826. Acesso em: 18 abril 2021

CASTRO-SÁNCHEZ, A.M. et al. Hydrotherapy for the Treatment of Pain in People with Multiple Sclerosis: A Randomized Controlled Trial. Evidence-Based Complementary and Alternative Medicine, vol.2012, pag.2-8, 2012. Disponível em: https://doi.org/10.1155/2012/473963. Acesso em: 08 abril 2021

COSTA, K.B. et al. Aspectos Respiratórios e a Fadiga em Pacientes com Esclerose Múltipla na Forma Remitente Recorrente. Revista Neurociências, São Paulo. vol.21, n.1, pag.36-42, 2013. Disponível em: phttp://dx.doi.org/10.4181/RNC.2013.21.649.7p. Acesso em: 13 mar. 2021

CHANG, Ya-Ju et al. Decreased central fatigue in multiple sclerosis patients after 8 weeks of surface functional electrical stimulation. Journal of Rehabilitation Research \& Development, vol.48, n.5, pag.555-564, 2011. Disponível em: DOI:10.1682/JRRD.2010.03.0038. Acesso em: 08 abril 2021

FESTUGATO, N. et al. Contribuições da ressonância magnética para auxílio ao 


\section{$2021 / 02$}

ISSN 2178-6925

diagnóstico de esclerose múltipla. Projeto de Intervenção Profissional II. Vol.4, pag.11, 2019. Caxias do Sul. Disponível em:

https://repositorio.ucs.br/11338/5859. Acesso em: 17 mar. 2021

GOMES, T.V. et al. Como os tratamentos alternativos e complementares para a esclerose múltipla contribuem para a qualidade de vida dos pacientes portadores da doença. Revista Eletrônica Acervo Científico, Caratinga- MG, vol.14, pag.5240, 2020. Disponível em: https://doi.org/10.25248/reac.e5240.2020. Acesso em: 08 mar. 2021

LIMA, N. et al. Efeitos do treinamento da musculatura respiratória em portadores de esclerose múltipla. Ensaios e Ciência: Ciências Biológicas, Agrárias e da Saúde.

Revistas Científicas da América Latina, Campo Grande, vol.15, n.1, pag.45-54, 2011. Disponível em: http://www.redalyc.org/articulo.oa?id=26019329004. Acesso em: 10 mar. 2021

MESQUITA, B.S. Análise da influência da abordagem fisioterapêutica sobre a fadiga em indivíduos com esclerose múltipla: uma revisão sistemática. Monografia (Bacharelado em Fisioterapia)-Universidade de Brasília, Brasília, pag.40, 2013. Disponível em: https://bdm.unb.br/handle/10483/6245. Acesso em: 18 mar. 2021

\section{MORAES, A.G. Efeitos da equoterapia no equilíbrio postural, mobilidade} funcional, marcha, fadiga e qualidade de vida em pessoas com esclerose múltipla. Tese (Doutorado em Educação Física)-Universidade de Brasília, Brasília, pag.178, 2020. Disponível em: https://repositorio.unb.br/handle/10482/40545. Acesso em: 26 mar. 2021

PEREIRA, G.C. et al. Combinações de Técnicas de Fisioterapia no Tratamento de Pacientes com Esclerose Múltipla: Série de Casos. Revista Neurociências, São Paulo, vol.20, n.4, pag.494-504, 2012. Disponível em: DOI: 
Revista Multidisciplinar do Nordeste Mineiro, v.2

\section{$2021 / 02$}

ISSN 2178-6925

https://doi.org/10.4181/RNC.2012.20.709.11p. Acesso em: 15 mar. 2021

PINA C. N.S. M. A intervenção da Fisioterapia na redução do risco de quedas na esclerose múltipla: revisão bibliográfica. Universidade Fernando Pessoa, Porto, pag.15-13, 2019. Disponível em: http://hdl.handle.net/10284/8805. Acesso em: 18 mar. 2021

SILVA, M.C.N. et al. Avaliação da qualidade de vida em portadores de esclerose múltipla: impacto da fadiga, ansiedade e depressão. Fisioterapia e Pesquisa. São Paulo, vol.26, n.4, pag.339-345, 2019. Disponível em: https://doi.org/10.1590/18092950/17005426042019. Acesso em: 20 mar. 2021

SCHIWE, D. et al. Fisioterapia em pacientes portadores de esclerose múltipla. Revista Saúde Integrada, vol.8, pag.15-16, 2015. Disponível em: https://core.ac.uk/download/pdf/229765591.pdf. Acesso em: 08 mar. 2021 